\title{
Recent Advances in Trigeminal Neuralgia and Its Management: A Narrative Review
}

\author{
Sourav Burman ${ }^{1} \quad$ Ankur Khandelwal ${ }^{2} \quad$ Arvind Chaturvedi $^{1}$ \\ ${ }^{1}$ Department of Neuroanaesthesiology and Critical Care, All India \\ Institute of Medical Sciences, New Delhi, India \\ 2Department of Anaesthesiology and Critical Care, Sharda University \\ School of Medical Sciences and Research, Greater Noida, \\ Uttar Pradesh, India

\begin{abstract}
Address for correspondence Arvind Chaturvedi, MD, Department of Neuroanaesthesiology and Critical Care, 6th Floor, C N Centre, All India Institute of Medical Sciences, Ansari Nagar, New Delhi 110029, India (e-mail: c.arvind61@yahoo.in).
\end{abstract}

J Neuroanaesthesiol Crit Care 2021;8:112-117.

\begin{abstract}
Keywords

- anticonvulsants

- facial pain

- neuromodulation

- pulsed radiofrequency

- trigeminal neuralgia

Trigeminal neuralgia (TN) is a chronic facial pain condition that affects one or more divisions of the trigeminal nerve (5th cranial nerve). It can be idiopathic, primary, or secondary. The cornerstone of the therapy has been antiepileptic medications, peripheral nerve blocks with various neurolytic agents, and surgical procedures. With the advent of newer technologies, minimally invasive neurolytic techniques like low-level laser therapy and ozone injection have revolutionized the management of TN. Novel drugs like vixotrigine and eslicarbazepine have been promising in reducing the frequency and severity of attacks. Inhaled carbon dioxide too has shown promising results in initial trials. Neuromodulation has given robust data in controlling neuralgic pain especially refractory to medical management. Pulsed radiofrequency has been used with increasing success and the side effects like dysesthesia and paresthesia are less. Cryotherapy, neural prolotherapy, and fiber knife techniques have helped us believe that TN can be controlled and cured. The need of the hour is to develop and explore newer modalities for trigeminal neuralgia treatment with minimum side effects. In this narrative review, we have tried to shed light into the newer modalities of treatment of TN along with new clinical classification for better disease recognition and management.
\end{abstract}

\section{Introduction}

Trigeminal neuralgia (TN) is a chronic facial pain condition that affects one or more divisions of the trigeminal nerve (5th cranial nerve), which carries sensation from face to brain. This nerve has three major branches: the ophthalmic nerve (V1), the maxillary nerve (V2), and the mandibular nerve (V3). Any single or multiple branches can contribute to the cause of the pain. The mandibular branch is the most frequently affected division. ${ }^{1}$ Pain occurs in areas supplied by the trigeminal nerve, including the cheek, jaw, teeth, gums, lips, or less often in the eyes and forehead. Pain is usually localized in the territory of the affected branch; however, it may migrate to other branches over time.

Published online May 24, 2021
DOI https://doi.org/

$10.1055 / \mathrm{s}-0041-1726152$

ISSN 2348-0548.
The International Association for Study of Pain (IASP) has defined TN as "sudden, usually unilateral, severe, brief, stabbing, recurrent episodes of pain in the distribution of one or more branches of the trigeminal nerve."2 A variety of triggers such as shaving, chewing, drinking, talking, smiling, brushing the teeth, washing the face, and encountering a breeze may initiate the pain of TN. Usually, pain resolves completely between the attacks. It usually does not occur when the person is asleep. Rapid spreading to other division, bilateral involvement, or simultaneous involvement of other nerve suggests a secondary disease such as multiple sclerosis (MS) or expanding cranial tumor. It is one of the most painful conditions and can result in depression and suicidal tendencies. ${ }^{3}$

(C) 2021. Indian Society of Neuroanaesthesiology and Critical Care.

This is an open access article published by Thieme under the terms of the Creative Commons Attribution-NonDerivative-NonCommercial-License, permitting copying and reproduction so long as the original work is given appropriate credit. Contents may not be used for commercial purposes, or adapted, remixed, transformed or built upon. (https://creativecommons.org/licenses/by-nc-nd/4.0/).

Thieme Medical and Scientific Publishers Pvt. Ltd. A-12, 2nd Floor, Sector 2, Noida-201301 UP, India 


\section{Demographics}

TN is a fairly common condition, with an incidence rate of 5.7 per 100,000 women and 2.5 per 100,000 men. The usual age range is around 50 to 70 years. TN is uncommon in young adults and rare in children. ${ }^{4}$

\section{Types of Trigeminal Neuralgia}

There are two varieties of TN: type 1 (classical TN) and type 2 (atypical TN). Occurrence of pain in type 1 is intermittent as described earlier. In type 2 , the pain is constant with less severity and is described as burning or pricking, rather than a shock. A subset of patients can progress from type 1 to type $2 \mathrm{TN}$ over time; thus, both types may coexist in the same person. A more recent and simple classification of TN has categorized TN into three types for simplicity of treatment options: possible TN, classical TN, and idiopathic TN $(\bullet \text { Fig. 1 })^{5}$

\section{Etiopathogenesis}

The exact etiology of TN is unknown, but mostly it is due to due to loss of the myelin around the trigeminal nerve. This may occur due to compression from a blood vessel (mostly superior cerebellar artery) at the root entry zone (REZ) near the foramen ovale. Other causes include multiple sclerosis, stroke, trauma, tumor, and arteriovenous malformation. There is evidence that TN precedes MS in patients. According to the American Society of Neurology, the pain felt in MS in young patients is mostly due to TN. Neuroimaging and surgical evidence suggests that neurovascular compression might act in concert with the pontine plaque through a double-crush mechanism. ${ }^{6}$ Established knowledge postulates that TN secondary to MS is associated with a pontine demyelinating plaque. The role of the pontine demyelinating plaque is also supported by functional neuroimaging studies

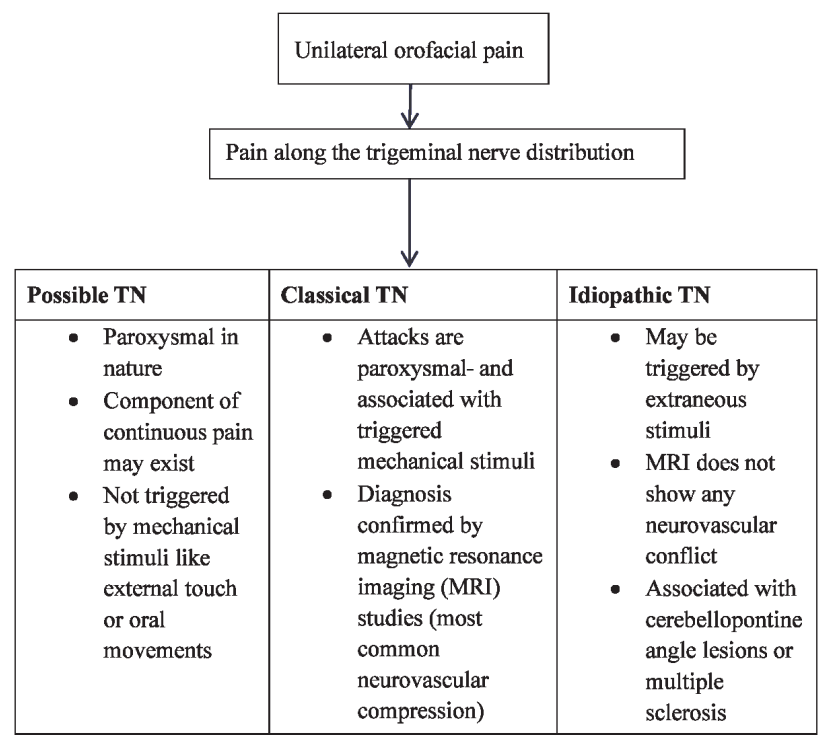

Fig. 1 New classification and diagnostic flowchart of trigeminal neuralgia (TN). showing that in patients with classical and idiopathic TN, tensor abnormalities are located in the cisternal and REZ segments of the trigeminal nerve, whereas in patients with TN secondary to MS the abnormalities are located in the pontine tract of the trigeminal nerve. ${ }^{7}$

\section{Theories Described for Trigeminal Neuralgia}

\section{Trigeminal Convergence Projection Theory}

The continuous nociceptive inputs that are received from the head and neck converge in the spinal trigeminal nucleus. The neurotransmitters released from the nucleus excite the second-order neurons which gives rise to a type of chronic neuropathic pain. ${ }^{8}$

\section{Bioresonance Hypothesis}

This is a new hypothesis which proposes that if the vibration frequency of a structure near the trigeminal nerve becomes close to its natural frequency, the resonance of the trigeminal nerve occurs. This can cause abnormal transmission and results in pain. ${ }^{9}$

\section{Ignition Hypothesis}

Injury to the trigeminal afferent neurons in the REZ makes the axons hyperexcitable and leads to synchronized discharge activity. ${ }^{10}$

\section{Diagnosis}

The diagnosis of TN is essentially clinical. Although such patients do not have any neurological deficit, quantitative sensory testing have shown subtle sensory abnormalities which may not be detected in routine clinical examination. Magnetic resonance imaging (MRI) is aimed to detect changes in trigeminal root and any neurovascular conflict, and to rule out secondary pathology. MRI sequences augmented by a three-dimensional gradient echo sequence such as fast inflow with steady-state precession (constructive interference in steady-state [CISS] sequence) or intravenous gadolinium-diethylene triamine pentaacetic acid (DTPA) can also improve visualization of the vascular compression around the trigeminal nerve root. ${ }^{11}$

\section{Treatment}

The anticonvulsant drug carbamazepine is the drug of choice in TN. The second drug of choice is oxcarbazepine. ${ }^{12}$ In a comparison between these two drugs, efficacy is very similar but tolerability is better with oxcarbazepine. Other drugs such as baclofen, lamotrigine, clonazepam, topiramate, phenytoin, gabapentin, pregabalin, and sodium valproate can be used. Polytherapy is useful when patients are unable to tolerate higher doses of carbamazepine. ${ }^{13}$ Opioids are considered ineffective against TN and, thus, should not be prescribed. A multidisciplinary approach using antidepressants and antianxiety drugs such as amitriptyline and duloxetine is needed for the management of emotional status. Acupuncture can be 
an option in the treatment of idiopathic TN due to its analgesic effect in both idiopathic TN and secondary myofascial pain associated with it. ${ }^{14}$

Peripheral nerve blocks using local anesthetic along with absolute alcohol or glycerol is also very effective in reducing pain. ${ }^{13,15}$ If nerve blocks are administered appropriately, patient may feel asymptomatic for few months to even years. ${ }^{16}$ It also reduces the number and doses of drugs. Small studies have shown that botulinum toxin type A (BTX-A) injections may reduce pain from $\mathrm{TN}$ in people who are no longer helped by medications. ${ }^{17} \mathrm{~A}$ recent meta-analysis showed a pooled reduction of pain by -3.009 points on a 0 to 10 verbal rating scale ( $95 \%$ confidence interval $[\mathrm{CI}]$, $p<0.001$ ) after treatment with BTX-A and confirmed moderate efficacy. ${ }^{18}$ However, more research needs to be done before this treatment is widely used for this condition.

Surgery is normally recommended only after medication has proved ineffective, or if side effects of medication are intolerable. Microvascular decompression is surgical treatment of choice in TN resistant to medical management, particularly, in young individuals. ${ }^{19}$ Patients with significant medical comorbidities are generally advised to undergo gamma knife radiosurgery, percutaneous balloon compression, glycerol rhizotomy, and radiofrequency thermocoagulation procedures. Partial sensory root sectioning is indicated in negative vessel explorations during surgery and large intraneural vein..$^{20}$ Endoscopic technique can be used alone for vascular decompression or as an adjuvant to microscope. ${ }^{21}$

\section{Recent Advances in Treatment Modalities}

After extensive literature search from popular databases like PubMed, Embase, Scopus, and Google Scholar, articles depicting newer treatment modalities were identified in the last 10 years. The keywords for search were "trigeminal neuralgia," "new modalities," and "future therapy." Those newer modalities which showed promising results in prospective randomized controlled trials and case-control studies were extracted and synthesized in this review. Data from recent meta-analysis were taken in case of certain treatment modalities that showed promising results.

\section{Recent Advances in Pharmacological Therapy}

The constant search for new drugs have led to the development of few recent medications that act via novel pathways for reducing the electrical activity of the already excited nerve.

1. Vixotrigine: Vixotrigine is a novel sodium channel blocker that preferentially targets higher frequencies and suppresses seizures or noxious stimuli. In an open-labeled study, vixotrigine $150 \mathrm{mg}$ administered thrice daily in patients with TN was compared with placebo and showed successful pain relief in the final week of therapy. ${ }^{22}$ The drug was administered for 21 days. There was a reduction in the number of paroxysms by $60 \%$ compared with only $12 \%$ in placebo, and pain severity decreased by $55 \%$ compared with placebo. The treatment failure rate was 33\% with this new drug and no serious adverse event was noted. A multicentric prospective phase III randomized controlled trial is already underway and its results will throw further light on this drug. ${ }^{23}$

2. Eslicarbazepine: It is a third-generation antiepileptic drug belonging to the dibenzepine group. The drug targets the voltage-gated sodium channels and is currently approved as adjunct therapy for focal seizures. In a recent open-labeled trial, eslicarbazepine was administered in a dose of 200 to $1200 \mathrm{mg} /$ day in patients suffering from TN. Around $88.9 \%$ patients had good pain relief but there was high incidence of side effects to the tune of $71 \%{ }^{24}$

3. Sumatriptan: It is a 5-hydroxytyptamine receptor $(1 \mathrm{~A} / \mathrm{B} / \mathrm{C})$ receptor blocker agonist. It has been used extensively in migraine and cluster headaches with good pain relief efficacy. The drug inhibits vasodilation and demyelination near the inflamed trigeminal nerve root. The drug comes in a formulation of tablets, nasal spray, or injections. Two randomized controlled trials tested the effect of subcutaneous injection of sumatriptan $3 \mathrm{mg}$ and the oral administration of $50 \mathrm{mg}$ twice daily. Fifteen minutes after injection of sumatriptan, the baseline pain scores decreased.25,26 After oral treatment, the visual analog score for pain also decreased significantly, and this effect persisted after treatment discontinuation for a week. The main side effect like dizziness and rebound headaches are common for which there is lack of adherence to therapy.

4. Intranasal carbon dioxide (CO2): $\mathrm{CO}_{2}$ has always been considered a pain modulator in hyperactive neurons. Recent studies have shown that $\mathrm{CO}_{2}$ is a nociceptive modulator of afferent active trigeminal neurons based on the hypothesis that $\mathrm{CO}_{2}$ causes a decreased mucosal $\mathrm{pH}$ and that in turn activates the nociceptive effect of primary trigeminal afferent neurons. ${ }^{27}$ A controlled, randomized, parallel-group study investigated the effects of intranasal $\mathrm{CO}_{2}$ on the transient receptor potential cation channel subfamily V member 1 (TRPV1)-mediated experimental trigeminal pain in healthy volunteers. Only mild modulatory effect of intranasal insufflation of $\mathrm{CO}_{2}$ at flow rates of $1 \mathrm{~L} / \mathrm{min}$ was found, but the clinical utility seemed limited since changes in pain ratings were therapeutically irrelevant. ${ }^{28}$ Hence, another phase 2 placebo-controlled trial was undertaken in which $\mathrm{CO}_{2}$ and placebo were administered in TN patients for 1 minute. All patients received three doses of $\mathrm{CO}_{2}$ and placebo each, and it was found that $\mathrm{CO}_{2}$ had improved effect on VAS scores. The trial is underway and its results are yet to be published (ClinicalTrials.gov identifier: NCT02473016). ${ }^{29}$

5. Calcium channel blockers (CCBs): Usually, in patients with continuous pain mediated by other pathophysiological mechanisms, a monotherapy with sodium channel blocker is not sufficient to control pain and other drugs are usually needed. CCBs and antidepressants have been advocated in the treatment of trigeminal neuralgia in patients not relieved by monotherapy with sodium 
channel blockers..$^{30}$ Thus, apart from few case reports or cohort studies there is very little evidence on management of continuous pain and more studies with CCBs are warranted.

6. Miscellaneous drugs: Various other medications like topical capsaicin, lignocaine, misoprostol, and intranasal lignocaine are available but their widespread use is not advocated at present. Misoprostol, a prostaglandin E1 analogue, showed efficacy in TN. Few studies reported the efficacy of misoprostol in a total of 27 patients with TN secondary to multiple sclerosis. ${ }^{31}$ However, there is insufficient evidence to support or refute the use of this drug in TN.

\section{Recent Advances in Nonpharmacological Therapy}

Nowadays apart from conventional RFA, new modalities utilizing reinforced $\mathrm{RF}$ doses for short periods called pulsed $\mathrm{RF}$ and other attenuated laser therapy are gaining importance. The list below enumerates the novel new therapeutic modalities.

1. Pulsed radiofrequency (PRF): PRF uses brief pulses of higher frequency alternate current to produce the same voltage or even higher fluctuations than during conventional radiofrequency (RF) treatment. PRF does not produce thermal lesions but there are microdamages within axonal microfilament and microtubules, especially in the pain-carrying $C$ fibers. ${ }^{32}$ Recent studies have shown that combination of both PRF and RF lesioning (RFL) has similar results in achieving a pain relief with lesser side effects than RFL alone. ${ }^{33}$ There is also less number of complications like anesthesia dolorosa and hyperesthesia with PRF. To achieve better results, PRF and RFL should be used in tandem rather than using these modalities separately.

2. Ozone injection around gasserian ganglion (OIAGG): Some newer studies have explored the role of OIAGG. In a multicentric retrospective study, the authors injected an ozone-oxygen mixture gas at a concentration of $30 \mu \mathrm{g} / \mathrm{mL}$ into the area around the gasserian ganglion performed under C-arm X-ray guidance. The results showed that pain relief rates at posttreatment, 6 months, 1 year, and 2 years after the procedure were $88.35 \%, 86.87 \%, 84.46 \%$, and $83.30 \%$, respectively $(p<0.05) .{ }^{34} \mathrm{~A}$ regression analysis found out that preoperative structural nerve damage was associated with less clinical effect or poor outcome. The study confirmed that OIAGG is a safe and effective modality for pain management in refractory TN.

3. Cryotherapy: To overcome the drawbacks of conventional cryotherapy such as incomplete pain relief and recurrence, few modifications have been suggested. These include (a) the use of a curved cryoprobe, (b) maintaining optimal temperature and pressure throughout the surgical procedure, (c) scoring of the epineurium, (d) application of petroleum jelly around the nerve before the introduction of the cryoprobe, and (e) delivery of three cycles of 3-minute freezing and 5-minute thawing to each nerve. ${ }^{35}$

In a study, Bansal et al showed that a closed curved cryoprobe tip when used with nitrous oxide at a temperature of $-98^{\circ} \mathrm{C}$ and a pressure of $70 \mathrm{~kg} / \mathrm{cm}^{2}$ or 100 psi provided excellent analgesia. Almost $48.97 \%$ patients had pain-free interval of 36 to 40 months. The side effect was loss of fine and crude sensation over face for 6 to 24 months. ${ }^{36}$

4. Neuromodulation: It is a new prospect in the management of TN patients, targeting either neural stimulation or inhibition to restore normal neurological function. Various neuromodulation techniques have been recently explored for the management of TN. These include transcranial magnetic stimulation, motor cortex stimulation, deep brain stimulation, spinal cord stimulation, transcutaneous electrical nerve stimulation, and peripheral nerve stimulation. A recent study is underway to establish the feasibility of using transcranial magnetic stimulation (TMS) for chronic orofacial pain in the interim period before surgery. Participants were randomized to either receive TMS or sham-TMS (a nontherapeutic TMS coil which sounds and feels similar to normal TMS), or standard treatment during the weeks of wait time before surgery. The sham TMS is a subtherapeutic level of magnetic stimulation which makes the same sound as normal TMS and causes a similar tingling of the skin. All study patients were asked to fill out an online survey about pain during different time points of the study. The complete results are not yet available, but preliminary results indicate that TMS, when applied to the head for a few minutes, has been shown to reduce pain in people with chronic orofacial pain of TN (ClinicalTrials.gov identifier: NCT04120129). ${ }^{37}$ Few studies have been conducted based on these techniques with variable success rate. ${ }^{38,39}$

5. Low level laser therapy (LLLT): LLLT uses a single wavelength light source and works on the principle that irradiation with monochromatic light may affect cell function. ${ }^{40}$ This technique involves irradiation of the region of interest followed by laser puncture at predetermined points along the course of the nerve. In a recent systematic review (8 randomized controlled trials, 2 prospective studies, and 3 case series) which evaluated the efficacy of LLLT for the therapeutic management of neuropathic orofacial pain, Pedro et al found a reduction in pain intensity in all studies (most of them significant). ${ }^{41}$ However, more quality studies assessing all outcome measures of chronic pain are warranted.

6. Carbon dioxide laser: $\mathrm{A} \mathrm{CO}_{2}$ laser is used to ablate the peripheral nerve in patients with drug refractory TN. Recently, it has been shown to reduce the pain scores in TN and persistence of pain relief till 12 months. ${ }^{42}$ The authors ablated peripheral nerves using low-power defocused mode; however, there was prolonged paresthesia of the affected nerves with this technique.

7. Neural prolotherapy: Neural prolotherapy has been described in relation to the management of TN. It is also known as perineural injection therapy (PIT) and is one of 
the latest advancements in regenerative medicine. First described by Dr. Paul Pybus and Dr. Roger Wyburn-Mason, PIT targets neurogenic inflammation in subcutaneous nerves that potentially generates pain. ${ }^{43}$ The technique involves injection of hypertonic dextrose saline with local anesthetics at the trigger points and usually requires multiple sittings. ${ }^{44}$

8. Nerve combing: Nerve combing, also called internal neurolysis, is a kind of surgical strategy that splits the branches of trigeminal nerve longitudinally using a special fiber knife based on preoperative pain locations and intraoperative finding. Jie et al studied 60 patients who achieved good pain relief following nerve combing. ${ }^{45}$ Nerve combing has a much higher pain relief rate in patients without vascular compression than those with vascular compression.

9. Complimentary medicine: Apart from standard conventional therapy there are several complimentary therapies that aid in pain relief of TN. These include standard acupuncture, electroacupuncture, and spinal regulation therapies. ${ }^{14}$ Other modalities of complimentary medicine include sound therapy; low-intensity and low-frequency acoustic ultrasound patch; and vitamin B, C, and biofeedback.

\section{Precautions in the Wake of COVID-19 Pandemic}

In the wake of the coronavirus disease 2019 (COVID-19) pandemic, certain precautions need to be taken while managing cases of TN. The standard operating protocols involve consultations in the outpatient department for those who are suffering from excruciating pain and not controlled with optimum dose of medications. Most of the patients with mild to moderate pain were treated with medicines and tele-consultations, which have become the norm in COVID-19 era. Most of the patients who are suffering from unbearable pain after maximal medication are advised for less invasive peripheral nerve blocks which can be done as a daycare procedure without the use of sedation or general anesthesia. The latest guidelines of European Society of Regional Anesthesia (ESRA) recommend that peripheral nerve blocks are non-aerosol-generating procedures and should be preferred than other invasive procedures. ${ }^{46}$ They have highlighted few precautions that need to be taken prior to such procedures:

1. Body temperature screening to be done and if temperature is more than $37.5^{\circ} \mathrm{C}$ then he/she should be directed to the flu clinic.

2. Rapid antigen testing or preferably RTPCR COVID-19 testing of all the patients before shifting to operation theatre complex.

3. Don appropriate personal protective equipment before doing the procedure, take extra time to doff, and use an observer.

4. All patients should wear proper universal precautions like surgical gown, mask, and gloves.

5. Ensure the use of plastic covers to protect ultrasound equipment.
6. Use minimal supplemental oxygen needed to maintain saturation.

7. Keep a high threshold for repeated blocks, as this may potentiate complications and increase exposure time.

\section{Conclusion}

There is a huge clinical burden of TN in our society. The age of presentation varies and hence there is a wide array of treatment options available. The treatment options need to be catered according to the age of the patient and the mode and type of presentation. The treatment modalities range from standard medical therapy and peripheral blocks to surgical procedures. Newer and novel techniques have arrived that show promising results. However, large randomized controlled trials are required for the validation of such techniques. The best technique should be simple, noninvasive, and achieved in a single setting with few recurrences, and provide long-term pain relief.

\section{Conflict of Interest}

None declared.

\section{References}

1 Bangash TH. Trigeminal neuralgia: frequency of occurrence in different nerve branches. Anesth Pain Med 2011;1(2):70-72

2 McMillan R. Trigeminal neuralgia-a debilitating facial pain. Rev Pain 2011;5(1):26-34

$3 \mathrm{Wu} \mathrm{TH}, \mathrm{Hu} \mathrm{LY}$, Lu T, et al. Risk of psychiatric disorders following trigeminal neuralgia: a nationwide population-based retrospective cohort study. J Headache Pain 2015;16:64

4 Hall GC, Carroll D, Parry D, McQuay HJ. Epidemiology and treatment of neuropathic pain: the UK primary care perspective. Pain 2006;122(1-2):156-162

5 Cruccu G, Finnerup NB, Jensen TS, et al. Trigeminal neuralgia: new classification and diagnostic grading for practice and research. Neurology 2016;87(2):220-228

6 Bonica JL, The Management of Pain. 2nd ed. Malvern, PA: Lea and Febiger; 1990:180

7 Di Stefano G, Truini A, Cruccu G. Current and innovative pharmacological options to treat typical and atypical trigeminal neuralgia. Drugs 2018;78(14):1433-1442

8 Chen DQ, DeSouza DD, Hayes DJ, Davis KD, O'Connor P, Hodaie M. Diffusivity signatures characterize trigeminal neuralgia associated with multiple sclerosis. MultScler 2016;22(1):51-63

9 Jia DZ, Li G. Bioresonance hypothesis: a new mechanism on the pathogenesis of trigeminal neuralgia. Med Hypotheses 2010;74(3):505-507

10 Devor M, Amir R, Rappaport ZH. Pathophysiology of trigeminal neuralgia: the ignition hypothesis. Clin J Pain 2002;18(1):4-13

11 Han-Bing S, Wei-Guo Z. Jun Z. Ning L, Jian-Kang S, Yu C. Predicting the outcome of microvascular decompression for trigeminal neuralgia using magnetic resonance tomographic angiography. J Neuroimaging 2010;20(4):345-349

12 Montano N, Conforti G, Di Bonaventura R, Meglio M, Fernandez E, Papacci F. Advances in diagnosis and treatment of trigeminal neuralgia. Ther Clin Risk Manag 2015;11:289-299

13 Hasan S. Trigeminal neuralgia: an overview of literature with emphasis on medical management. Int Res J Pharma. 2012;3:235-238

14 Mishra RK, Rath GP, Complimentary medicine in trigeminal neuralgia. In: Rath GP, ed. Handbook of Trigeminal Neuralgia. Singapore: Springer Nature; 2019 207-213 
15 Perloff MD, Chung JS. Urgent care peripheral nerve blocks for refractory trigeminal neuralgia. Am J Emerg Med 2018;36(11):2058-2060

16 Han KR, Chae YJ, Lee JD, Kim C. Trigeminal nerve block with alcohol for medically intractable classic trigeminal neuralgia: long-term clinical effectiveness on pain. Int J Med Sci 2017;14(1):29-36

17 Zhang H, Lian Y, Ma Y, et al. Two doses of botulinum toxin type A for the treatment of trigeminal neuralgia: observation of therapeutic effect from a randomized, double-blind, placebo-controlled trial. J Headache Pain 2014;15:65

18 Fischoff DK, Spivakovsky S. Botulinum toxin for facial neuralgia. Evid Based Dent 2018;19(2):57-58

19 Gu W, Zhao W. Microvascular decompression for recurrent trigeminal neuralgia. J Clin Neurosci 2014;21(9):1549-1553

20 Abhinav K, Love S, Kalantzis G, Coakham HB, Patel NK. Clinicopathological review of patients with and without multiple sclerosis treated by partial sensory rhizotomy for medically refractory trigeminal neuralgia: a 12-year retrospective study. ClinNeurolNeurosurg 2012;114(4):361-365

21 Zagzoog N, Attar A, Takroni R, Alotaibi MB, Reddy K. Endoscopic versus open microvascular decompression for trigeminal neuralgia: a systematic review and comparative meta-analysis. J Neurosurg 2018;7:1-9

22 Hesselink JMK. Moving targets in sodium channel blocker development: the case of raxatrigine: from a central NaV1.3 blocker via a peripheral NaV1.7 blocker to a less selective sodium channel blocker. J Med Therap 2017;1:1-3

23 Obermann M. Recent advances in understanding/managing trigeminal neuralgia. F1000 Res 2019;8:F1000

24 Sanchez-Larsen A, Sopelana D, Diaz-Maroto I, et al. Assessment of efficacy and safety of eslicarbazepine acetate for the treatment of trigeminal neuralgia. Eur J Pain 2018;22(6):1080-1087

25 Moore D, Chong MS, Shetty A, Zakrzewska JM. A systematic review of rescue analgesic strategies in acute exacerbations of primary trigeminal neuralgia. $\mathrm{Br}$ J Anaesth 2019;123(2):e385-e396

26 Kanai A, Suzuki A, Osawa S, Hoka S. Sumatriptan alleviates pain in patients with trigeminal neuralgia. Clin J Pain 2006;22(8):677-680

27 Vause C, Bowen E, Spierings E, Durham P. Effect of carbon dioxide on calcitonin gene-related peptide secretion from trigeminal neurons. Headache 2007;47(10):1385-1397

28 Jürgens TP, Reetz R, May A. No relevant modulation of TRPV1-mediated trigeminal pain by intranasal carbon dioxide in healthy humans. J Headache Pain 2013;14:33

29 ClinicalTrials.gov. Nasal carbon dioxide for the symptomatic treatment of classical trigeminal neuralgia. Available at: https://clinicaltrials.gov/ct2/show/record/NCT02473016. Published June 16, 2015. Accessed December 28, 2020

30 Patel R, Montagut-Bordas C, Dickenson AH. Calcium channel modulation as a target in chronic pain control. $\mathrm{Br}$ J Pharmacol 2018;175(12):2173-2184

31 Pfau G, Brinkers M, Treuheit T, Kretzschmar M, Sentürk M, Hachenberg T. Misoprostol as a therapeutic option for trigeminal neuralgia in patients with multiple sclerosis. Pain Med 2012;13(10):1377-1378

32 Bogduk N. Pulsed radiofrequency. Pain Med 2006;7(5):396-407

33 Liao C, Visocchi M, Yang M, Liu P, Li S, Zhang W. Pulsed radiofrequency: a management option for recurrent trigeminal neuralgia following radiofrequency thermocoagulation. World Neurosurg 2017;97:760.e5-760.e7

34 Gao L, Chen RW, Williams JP, et al. Efficacy and safety of percutaneous ozone injection around gasserian ganglion for the treatment of trigeminal neuralgia: a multicenter retrospective study. J Pain Res 2020;13:927-936

35 Zhou L, Shao Z, Ou S. Cryoanalgesia: electrophysiology at different temperatures. Cryobiology 2003;46(1):26-32

36 Bansal V, Mowar A, Dubey P, Gupta S. Role of cryotherapy in trigeminal neuralgia with certain modifications: a long-term prospective study. Oral Surg Oral Med Oral Pathol Oral Radiol 2020;129(4):290-295

37 ClinicalTrials.gov. Transcranial magnetic stimulation (tms) for trigeminal neuralgia (TGN). Available at: https://clinicaltrials.gov/ct2/show/study/NCT04120129. Published October 9, 2019. Accessed December 28, 2020

38 Ben-Haim S, Mirzadeh Z, Rosenberg WS. Deep brain stimulation for intractable neuropathic facial pain. Neurosurg Focus 2018;45(2):E15

39 Gibson W, Wand BM, O'Connell NE. Transcutaneous electrical nerve stimulation (TENS) for neuropathic pain in adults. Cochrane Database Syst Rev 2017;9:CD011976

40 Samosiuk IZ, Kozhanova AK, Samosiuk NI. Fiziopunkturnaia terapiia nevralgii troĭnichnogo nerva [Physiopuncture therapy of trigeminal neuralgia]. Vopr Kurortol Fizioter Lech Fiz Kult 2000;(6):29-32

41 de Pedro M, López-Pintor RM, de la Hoz-Aizpurua JL, Casañas E, Hernández G. Efficacy of low-level laser therapy for the therapeutic management of neuropathic orofacial pain: a systematic review. J Oral Facial Pain Headache 2020;34(1):13-30

42 Sessirisombat S. Carbon-dioxide laser in the treatment of trigeminal neuralgia: a preliminary study. J Interdiscipl Med Dent Sci 2017;5:208

43 Reeves KD, Lyftogt J, Prolotherapy: regenerative injection therapy. In: Waldman SD, ed. Pain Management. 2nd ed. Philadelphia, PA: Saunders (Elsevier); 2011:1027-44

44 Khandelwal A, Rath GP, Neural prolotherapy for trigeminal neuralgia. In: Rath GP, ed. Handbook of Trigeminal Neuralgia. Singapore: Springer Nature; 2019:201-205

45 Jie H, Xuanchen Z, Deheng L, et al. The long-term outcome of nerve combing for trigeminal neuralgia. Acta Neurochir (Wien) 2013;155(9):1703-1708

46 Uppal V, Sondekoppam RV, Lobo CA, Kolli S, Kalagara HKP. Practice recommendations on neuraxial anesthesia and peripheral blocks during the COVID-19 pandemic. Available at: www.asra.com/covid-19/raguidance. Published March 27, 2020. Accessed December 28, 2020 